\title{
Siderophore production by Gram-negative rods isolated from human polymicrobial infections
}

\author{
JOANNA MOKRACKA, EWA CICHOSZEWSKA and ADAM KAZNOWSKI \\ Department of Microbiology, Faculty of Biology, Adam Mickiewicz University, Umultowska 89, \\ 61-614 Poznań, Poland. \\ Corresponding author: Adam Kaznowski, akazn@amu.edu.pl
}

(Received on 23 April 2010; Accepted on 15 March 2011)

\begin{abstract}
A total of 137 strains isolated from 67 mixed bacterial infections were examined for production and interchangeability of siderophores. The isolates comprised 109 strains belonging to 15 species of Enterobacteriaceae and 28 isolates of 6 species of non-fermenting rods. In 36 mixed infections (53.7\%), the strains secreted siderophores of the same type. This concerned mostly strains belonging to the Enterobacteriaceae $(46.3 \%)$, which produced enterobactin. We selected 37 pairs of strains that produced different siderophores. The strains examined were not able to use siderophores produced by the other isolate of the pair, except for 3 strains of Pseudomonas aeruginosa that used chelators excreted by enterobactinproducing $E$. coli. Our research indicates that in mixed polymicrobial infections the interchangeability of siderophores is possible, although it seems to be rare. More common is the production and secretion of the same chelator by strains participating in one infection, which definitely leads to an increase in the amount of iron chelator at the site of infection and, consequently, may enhance the virulence potential of bacteria, as the amount of siderophore seems to be directly related to the pathogenicity of a strain.
\end{abstract}

Keywords: iron chelators, mixed bacterial infection, Gram-negative rods

\section{INTRODUCTION}

Mixed bacterial infections comprise 2 or more strains involved in a particular type of synergism, in which each organism may derive essential growth factors from the other. Polymicrobial infections are more pathogenic for experimental animals than those involving a single organism (MELENEY 1931).

One of the crucial steps in establishing an infection is the availability of iron. Its concentration in human blood and other tissues is extremely low, while most bacteria require $10^{-6} \mathrm{M}$ (WEINBERG 1978; ANDREWs et al. 2003). A lack of available iron can be bacteriostatic or even lethal for microorganisms. To overcome the lack of available iron, bacterial strains use several strategies to acquire it from mammalian hosts. The major one is a biosynthesis of siderophores and their cognate transport systems residing 
in the inner and outer membrane. Ferri-siderophore $\left(\mathrm{Fe}^{3+}\right.$-loaded) is selectively recognized and bound by receptors on the cell surface and then transported into the cell (FARALDO-GómEZ \& SANSOM 2003). Iron sequestration involving siderophores is a recognized factor essential for bacterial pathogenicity. Deletion of siderophore biosynthetic genes leads to a loss of pathogenicity in a mouse infection model, and also affects maturation of biofilms, surface motility, activation of exotoxins and synthesis of other virulence factors (VoKes et al. 1999; VisCA et al. 2007; Mossialos \& AMOUTZIAS 2008). Additionally, the presence of siderophores in the human host may play a role in activating interleukin-8 secretion and hypoxia-independent HIF-1 (hypoxia inducible factor-1), a key transcriptional activator during hypoxia, which appears to represent the host defense mechanism (NELSON et al. 2007; HARTMANN et al. 2008). Polymicrobial infections have never been examined for siderophore interchangeability of the participating strains. The cross-feeding, where one strain partially degrades the primary energy resource and excretes an intermediate that is used as an energy resource by a second strain, has been proved in populations of bacteria. As far as the acquisition of iron is considered, a strain involved in polymicrobial infection could benefit from siderophore potential of the other. So far, there is evidence that a siderophore produced by one strain can induce gene expression in another isolate participating in the infection (WEAVER \& KOLTER 2004). The aim of this study was to determine the type of siderophore excreted by strains taking part in mixed infections of humans and to analyze if the strains participating in an infection can take advantage of the siderophore produced by the co-infecting isolate.

\section{MATERIALS AND METHODS}

\section{Clinical specimens}

A total of 137 isolates comprising 109 strains belonging to 15 species of Enterobacteriaceae, and 28 isolates of 6 species of non-fermenting rods were included in this study. The isolates of Enterobacteriaceae comprised Escherichia coli (38 strains), Klebsiella pneumoniae (30 strains), K. oxytoca (3 strains), K. ornithinolytica (1 strain), Enterobacter cloacae (9 strains), E. sakazakii (2 strains), Proteus mirabilis (15 strains), P. vulgaris (1 strain), Citrobacter freundii (3 strains), Serratia odorifera (2 strains), S. ficaria (1 strain), S. marcescens (1 strain), S. liquefaciens (1 strain), Hafnia alvei (1 strain), and Morganella morganii (1 strain). Non-fermenting rods belonged to Pseudomonas aeruginosa (19 strains), Acinetobacter baumannii (4 strains), A. lwoffii (1 strain), Brevundimonas vesicularis (2 strains), Burkholderia cepacia (1 strain), and Stenotrophomonas maltophilia (1 strain). The strains were cultured from various samples (urine, pharyngeal swabs, wounds, tracheostomy tubes, bronchial tubes, pleural cavity fluids, sputum from peritoneal cavity, blood and abscess) from inpatients at a hospital in Poznań, Poland. They were isolated from polymicrobial infections in which two (64 cases) or three (3 cases) bacterial strains were cultured. The organisms were identified with API 20E, API 20NE and ID 32GN (bioMérieux, France). The strains and their sources are listed in Table 1. 


\section{Siderophore assays}

Bacterial strains were grown in $\mathrm{M} 9$ mineral medium containing $0.3 \mathrm{~g}$ of $\mathrm{KH}_{2} \mathrm{PO}_{4}$, $5 \mathrm{~g}$ of $\mathrm{NaCl}, 1 \mathrm{~g}$ of $\mathrm{NH}_{4} \mathrm{Cl}, 0.0015 \mathrm{~g}$ of $\mathrm{CaCl}_{2}, 0.024 \mathrm{~g}$ of $\mathrm{MgSO}_{4} \times 7 \mathrm{H}_{2} \mathrm{O}, 2 \mathrm{~g}$ of casamino acids, $2 \mathrm{~g}$ of succinic acid, $1000 \mathrm{ml}$ of deionized water, $\mathrm{pH}$ 7.2. The medium was deferrated with Chelex 100 (BioRad) for iron-deficient conditions. Overnight cultures were centrifuged at $3000 \mathrm{~g}$, and the supernatants were analyzed. Bacteria were screened for production of siderophores by the standard method of ScHWYN \& NeILANDS (1987) with chrome azurol S (CAS) assay solution. Hydroxamate and catecholate chelators were assessed according to the methods of CSÁKY (1948) and ARNow (1937), respectively.

\section{Absorption spectrum}

Bacteria of the genus Pseudomonas may produce fluorescent siderophores. The siderophores were identified in cell-free supernatants. Overnight cultures of Pseudomonas aeruginosa were centrifuged at $3000 \mathrm{~g}$, and the absorbance spectra of supernatants were analyzed according to Manninen \& Matilla-SAndholm (1994).

\section{Minimal Inhibitory Concentration (MIC) of alpha, alpha'-dipyridyl}

In order to estimate the MIC of alpha, alpha'-dipyridyl (Sigma), the strains were grown overnight in Brain Heart Infusion. Melted Luria agar (LA), with various concentrations of alpha, alpha'-dipyridyl, was cooled and mixed with $10^{2} \mathrm{cfu} / \mathrm{ml}$ of the tested strain and poured onto plates. The plates were incubated for $18 \mathrm{~h}$ at $37^{\circ} \mathrm{C}$. The lowest concentration of alpha, alpha'-dipyridyl that inhibited the growth of a strain was determined as the MIC value.

\section{Cross-feeding assays}

Siderophores were determined by cross-feeding assays, which test the ability of bacteria to promote the growth of siderophore-deficient receptor-proficient indicator strains grown under iron starvation. Shigella flexneri SA 255 (enterobactin indicator), Escherichia coli LG 1522 (aerobactin and rhodotorulic acid indicator), Microbacterium flavescens JG-9 (the indicator strain for hydroxamate siderophores distinct from aerobactin: coprogen, ferrichrome, rhodotorulic acid, and ferroxiamine B), Morganella morganii SBK 3 (rhizoferrin and $\alpha$-keto acid indicator) and Yersinia enterocolitica 5030 (yersiniabactin indicator) (REISSBRODT \& RABSCH 1988; RABSCH \& Winkelmann 1991; HaAg et al. 1993).

\section{PCR analyses}

The presence of yersiniabactin biosynthetic genes was confirmed by PCR analysis with primers irp1, irp2. All PCR reagents were purchased from Novazym (Poland). Conditions of PCR amplifications, including the sequences of primers, were previously published (KARCH et al. 1999). The PCR products were separated in $1.5 \%$ agarose gel. All experiments were performed in triplicate. 


\section{Bioassay for interchangeability of siderophores}

Inoculum of $10^{2} \mathrm{cfu} / \mathrm{ml}$ of one strain of the pair (recipient), was mixed with LA containing alpha, alpha'-dipyridyl at MIC concentration and used as a lawn. The second strain of the pair (donor) was seeded punctually onto the surface of the agar and the plate was incubated for $18 \mathrm{~h}$ at $37^{\circ} \mathrm{C}$. After incubation the plates were observed for growth of the recipient strain around the donor strain.

\section{Statistical analysis}

The Monte Carlo permutation test was used to calculate a $P$ value associated with selection of co-infecting strains upon the type of siderophore produced (HopE 1968).

\section{RESULTS AND DISCUSSION}

We determined the ability of extraintestinal strains of Gram-negative rods to employ different siderophore-mediated strategies of iron acquisition and the possibility of siderophore interchangeability between strains involved in one infection. We analyzed in total 67 cases of polymicrobial infection. Among them, 44 cases comprised exclusively strains of Enterobacteriaceae, whereas 18 cases involved both the Enterobacteriaceae and non-fermentative Gram-negative rods. Five cases of infections were connected exclusively with non-fermentative Gram-negative rods (Table 1).

All strains examined in this study produced iron chelators when assessed in the universal CAS assay. Further analyses included estimation of the chemical class of chelators and determination of the type of chelator in cross-feeding assays with indicator strains. Ninety-five strains $(69.3 \%)$ excreted catechol chelators, while 74 strains (54\%) produced hydroxamate ligands (Table 1$)$.

Biological assays with indicator strains deficient in siderophore synthesis but capable of acquiring exogenous iron chelators indicated that 91 strains (66.4\%) excreted the catecholate siderophore enterobactin (Table 1). The strains belonged mostly to the Enterobacteriaceae ( 90 isolates), as enterobactin is the prevalent siderophore produced by bacteria of this family (OKEKE et al. 2004). Non-fermentative rods did not produce enterobactin except one strain of Stenotrophomonas maltophilia, isolated together with Acinetobacter baumannii from a wound infection.

Thirteen isolates $(9.5 \%)$ produced the hydroxamate siderophore aerobactin. Aerobactin producers included mostly E. coli ( 7 strains), isolated from pharyngeal swab (3 strains), urine (2 strains), wound ( 2 strain) and tracheostomy tube (1 strain), and $E$. cloacae (3 strains) originating from wound ( 2 strains) and bronchial tubes (1 strain). The other 2 species were $K$. pneumoniae and $S$. odorifera, isolated from urine and pharyngeal swab, respectively. The production of aerobactin was not directly related to the source of an isolate, although most aerobactin producers were obtained from wounds, pharyngeal swabs, and urine. In cross-feeding assays, many more strains expressed the production of hydroxamate siderophores other than aerobactin, namely rhodotorulic acid, coprogen and/or ferroxiamine B. Those kinds of chelators were excreted by as many as 42 isolates (30.7\%) belonging to Pseudomonas sp. 


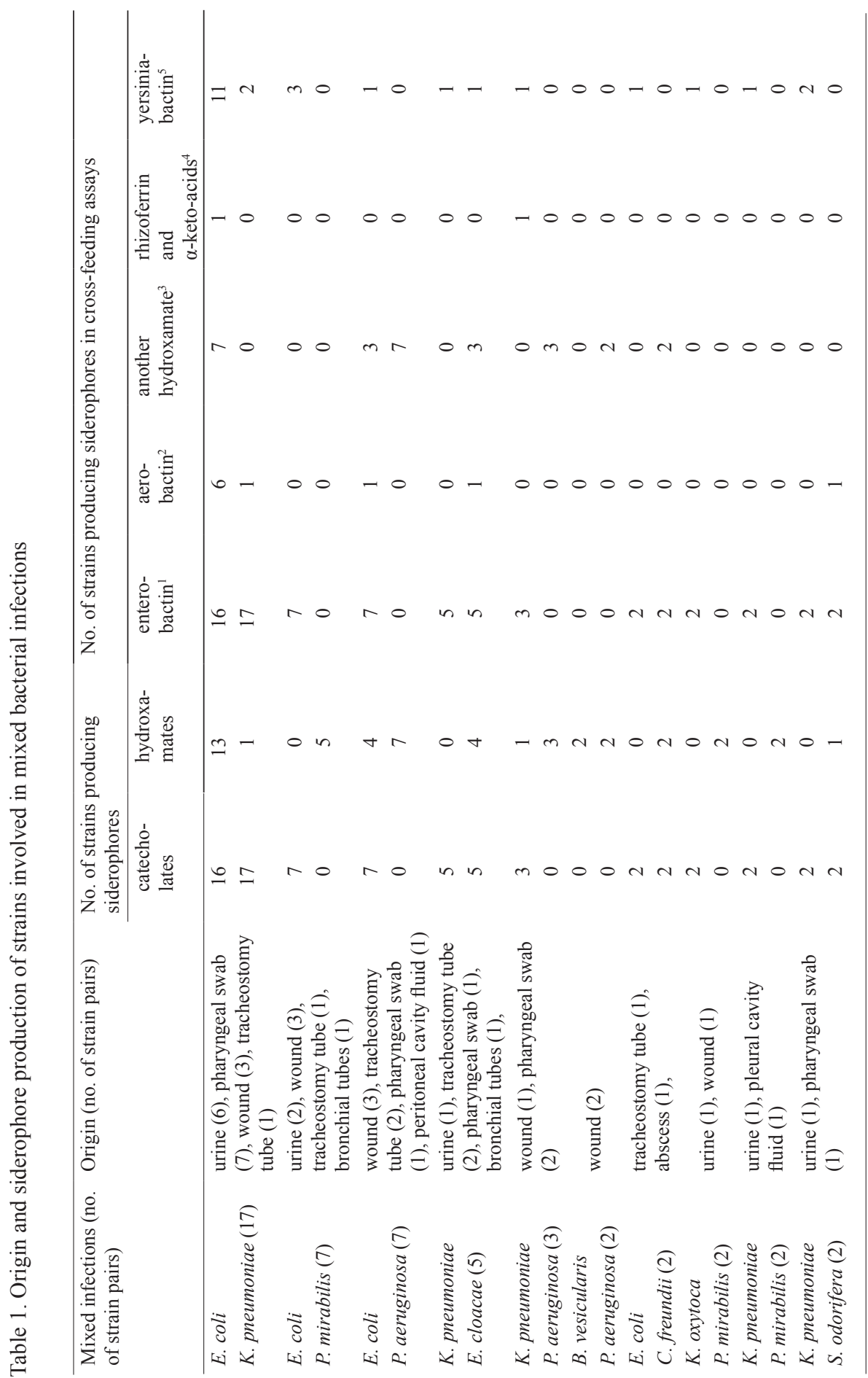




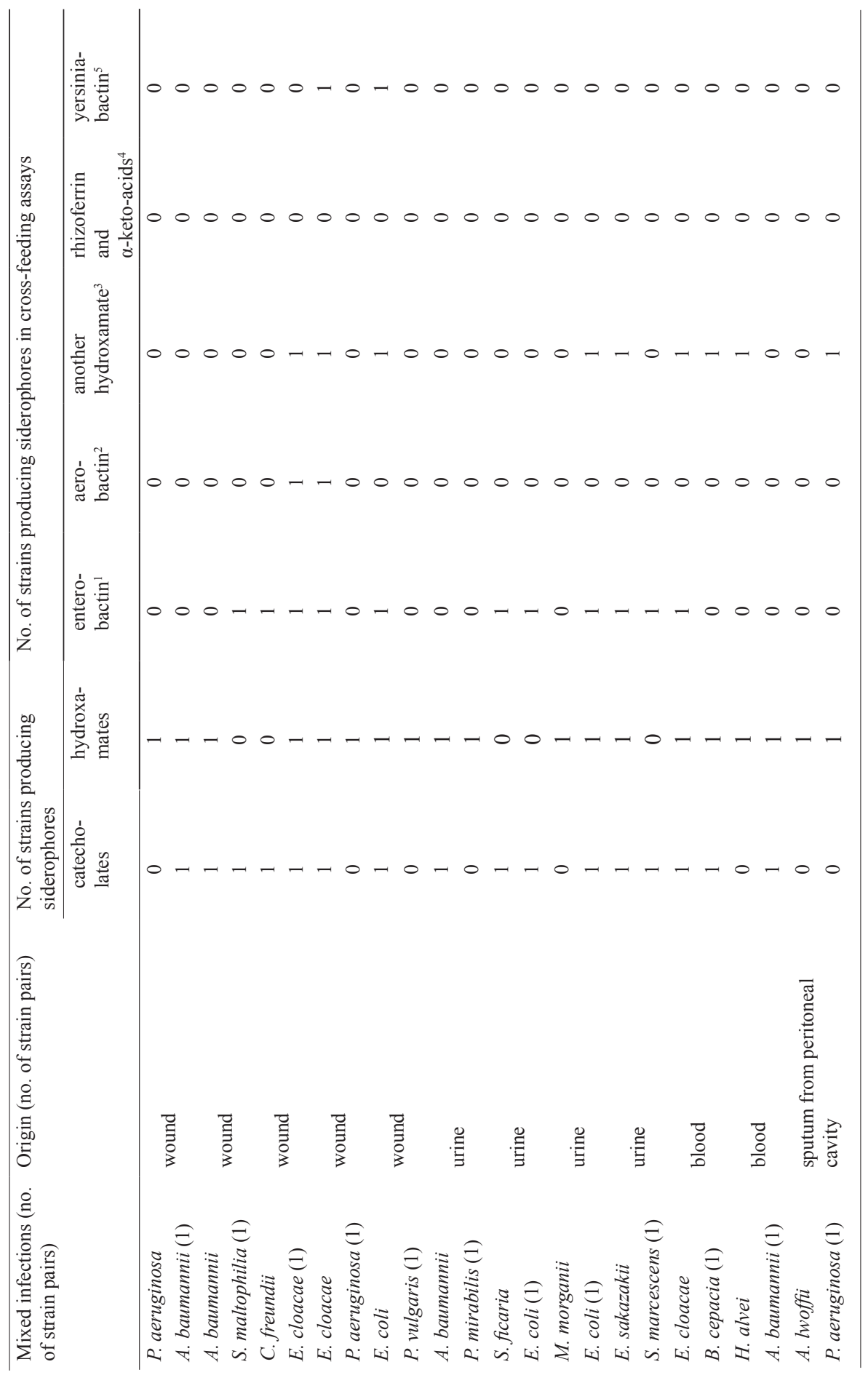




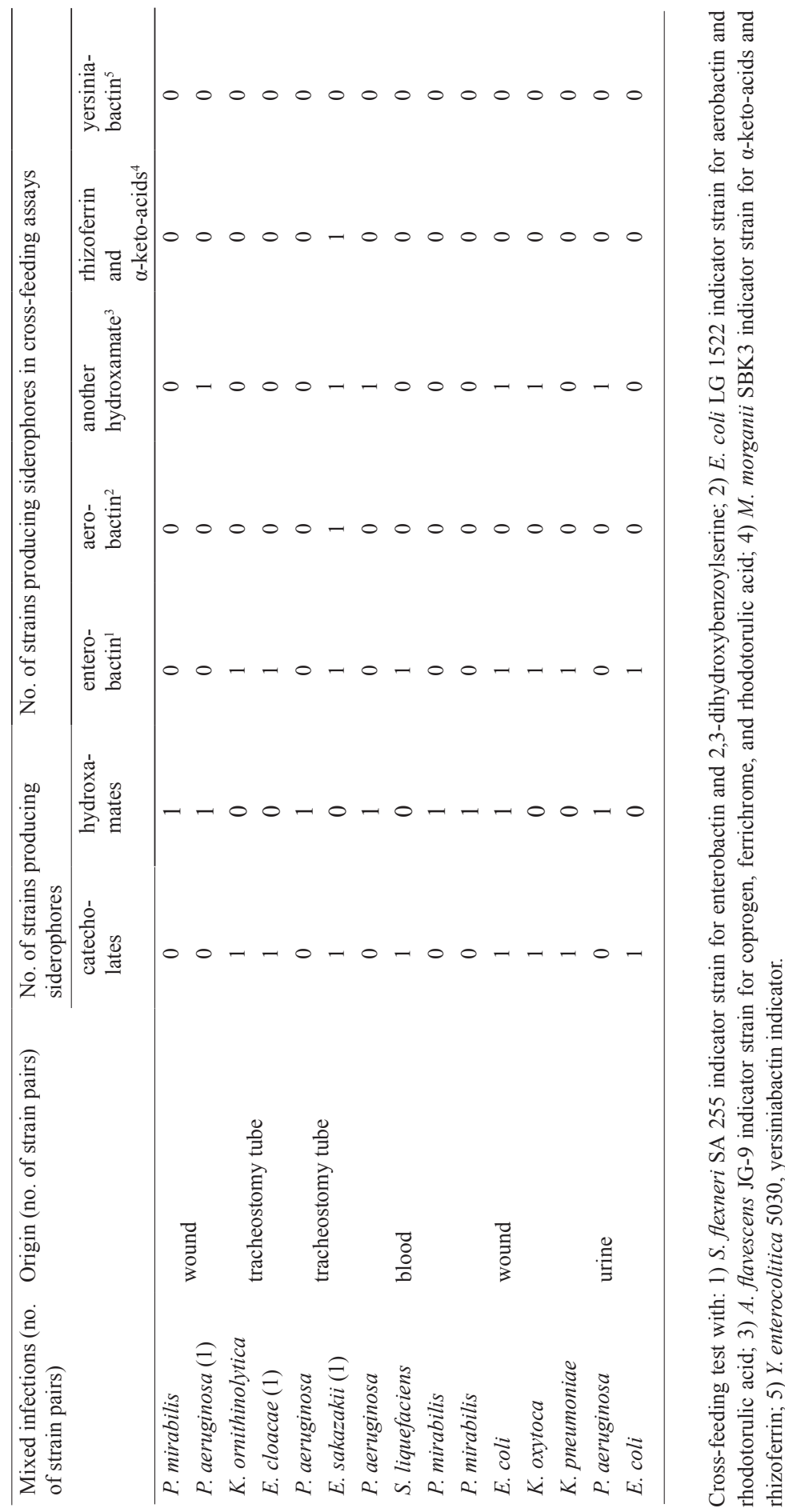


(16 strains), E. coli (13 strains), Enterobacter sp. (8 strains), C. freundii (2 strains), B. cepacia (1 strain), H. alvei (1 strain), and $K$. oxytoca (1 strain).

Twenty-seven strains were able to cross-feed indicator strain Yersinia enterocolitica 5030, which indicates yersiniabactin production. Astoundingly, yersiniabactin (a siderophore originally described in pathogenic species of Yersinia, being a part of the high-pathogenicity island with a proven impact on their pathogenicity) was excreted by as many as 27 strains (20\%). The biological assays were confirmed by gene amplifications. Yersiniabactin producers comprised mostly E. coli (17) and K. pneumoniae (7) strains, one strain of K. oxytoca, and 2 of E. cloacae isolated from pharyngeal swab, urine, tracheostomy tubes and wounds. The yersiniabactin system of iron acquisition is the third most prevalent among the Enterobacteriaceae (LAWLOR et al. 2007). In this research, the yersiniabactin system appeared to be more dominant than the aerobactin system, which indicates its potential role in virulence and probability of horizontal transfer of the high-pathogenicity island. The yersiniabactin system is a proven virulence factor in extraintestinal infection of $E$. coli and $K$. pneumoniae pulmonary infections (SCHUBERT et al. 2002; LAWLOR et al. 2007).

In cross-feeding assays testing Proteus sp. (16 strains) and Morganella morganii (1 strain), we did not identify the chelator. However, in the Csáky test, 14 Proteus sp. strains $(87.5 \%)$ revealed production of hydroxamate ligands, whereas $M$. morganii secreted a catechol chelator detected in the Arnow test.

We examined 19 strains of Pseudomonas sp. In biological assays most of the isolates $(84.2 \%)$ produced hydroxamates other than aerobactin, i.e. coprogen, ferrichrome, rhodotorulic acid or ferroxiamine B (Table 1). Additionally, we analyzed spectrophotometrically cell-free supernatants of pseudomonads and in all cases identified chelators with maximum absorption at $\lambda=410 \mathrm{~nm}$, which is typical to pyoverdine, a fluorescent siderophore produced by Pseudomonas sp. (MEYER et al. 2002). Pyoverdine can acquire the iron bound to transferrin and lactoferrin (XIAO \& KISAALITA 1997). The other Gram-negative non-fermentative rods, namely, one B. cepacia strain and one strain of $S$. maltophilia, excreted siderophores with both catechol and hydroxamate moieties. In biological cross-feeding assays, S. maltophilia revealed the production of enterobactin. Strains of the genus Acinetobacter were negative in cross-feeding assays, i.e. they did not produce any of the tested siderophores, but in the universal CAS assay they showed the production of iron chelators, and in Csaky and Arnow tests they secreted catechol and hydroxamate ligands that appeared to be acinetobactin - a chelator with catechol and hydroxamate functional groups (YАмамото et al. 1994; Dorsey et al. 2004).

In 36 cases (including 2 cases with 3 strains participating in infection) of mixed infections $(53.7 \%)$, the strains secreted a siderophore of the same type. This applies mostly to strains belonging to the Enterobacteriaceae (30 pairs, 44.7\%), which produced enterobactin (Table 1). In one case, both E. coli and K. pneumoniae strains isolated from urine produced simultaneously enterobactin and aerobactin, and in one case of $K$. pneumoniae and $E$. coli urinary tract infection, both strains secreted yersiniabactin. The remaining 5 pairs comprised strains of $E$. coli and $P$. aeruginosa (3 pairs), and E. cloacae and B. cepacia, all of which produced hydroxamates other than aerobactin. To establish the exact role of such a "mutual" siderophore, we must take into 
consideration the site of infection and the type of siderophore. Aerobactin, which is a powerful siderophore and contributes to the virulence of some bacterial species (Podschun \& UlLmann 1998), was rarely excreted by both strains participating in the infection. E. coli and K. pneumoniae isolated from urine were the only example. In most cases in which strains produced the same chelator, they excreted enterobactin, which has a higher affinity for iron than human iron-binding proteins. Nonetheless, its role in bacterial virulence is not clear. For example, the mammalian protein siderocalin binds enterobactin, making it ineffective in bacterial iron acquisition (NELSON et al. 2007).

Regardless of that, we can draw a conclusion that if the strains participating in one infection produce the same type of siderophore, it leads to an increase in the amount of iron chelator at the site of infection, and, consequently, may enhance the virulence potential of bacteria, as the amount of siderophore present seems to be directly related to the pathogenicity of a strain. That was the case in an infection caused by Staphylococcus aureus, whose virulence was higher when it produced more siderophores (RóżALSKA et al. 1998). In mixed infections in which both strains excrete the same siderophore, especially in a situation when one strain produces definitely more chelators, it can enhance the possibility of colonization by the second, otherwise less potent strain.

To check the possibility of using exogenous chelator produced by the coinfecting strain, we selected 37 pairs in which strains produced different siderophores (Table 1). That concerned one group created by strains of $P$. mirabilis isolated together with E. coli (8), K. pneumoniae (2), K. oxytoca (3), A. baumannii (1), S. liquefaciens (1), and one pair of $P$. vulgaris and $E$. coli, and a second group created by $P$. aeruginosa isolated together with: E. coli (5), K. pneumoniae (4), P. mirabilis (2), B. vesicularis (2), E. cloacae (1), E. sakazakii (1), A. lwoffii (1), A. baumannii (1), and S. liquefaciens (1). Moreover, we analyzed one pair of S. maltophilia and A. baumannii, one pair of H. alvei and A. baumannii, and one pair of M. morganii and E. coli.

For each strain, we determined the MIC of alpha, alpha'-dipyridyl and analyzed the possibility of mutual cross-feeding in bioassays for interchangeability of siderophores.

In most cases the strains were not able to cross-feed one another. However, we observed that 3 strains of $P$. aeruginosa could use chelators excreted by $E$. coli strains participating in the same infection. The strains were isolated from a wound, tracheostomy tube, and peritoneal cavity fluid. Strains of the genus Pseudomonas excreted coprogen and/or ferrichrome, rhodotorulic acid, and ferroxiamine B. Moreover, they produced pyoverdine, detected by spectrophotometry. E. coli strains which, together with Pseudomonas sp., participated in these infections, produced enterobactin. E. coli strains could not be cross-fed by isolates of Pseudomonas sp., whereas strains of $P$. aeruginosa could use exogenous siderophore produced by $E$. coli. This suggests the existence of a receptor and transport system for other than endogenously produced siderophores. In earlier research with exogenously added chelators, such as enterobactin, aerobactin, ferroxiamine B, and pyochelin, some strains of Pseudomonas sp. were able to use them (Dean \& Poole 1993; BudziKiewicz 1997). As enterobactin has the highest affinity for iron, the ability of pseudomonads to use this chelator may 
enhance the possibility of surviving in the tissues where iron availability is extremely low and where it is tightly bound to lactoferrin and/or transferrin.

Our research indicates that in mixed polymicrobial infections, the interchangeability of siderophores is rare. A more common situation is the production and secretion of the same chelator by strains participating in one infection. Irrespective of the kind and amount of chelator, it remains clear that the combination of strains producing the same type of siderophore or being able to use exogenous chelators can significantly enhance the chances of acquiring iron in the environment where its concentration is extremely low. However, according to the Monte Carlo permutation test, we cannot reject the null hypothesis that the observed fraction of co-infection by strains producing the same siderophore can be explained by chance $(P=0.89)$.

Another aspect of potential importance is the opportunity for horizontal gene transfer of iron-sequestering systems, in the context of co-infection. The presence of yersiniabactin locus on the potentially mobile high-pathogenicity island in $20 \%$ of strains may thus hint that co-existence of 2 strains or more in one infection site enhances the probability of virulence gene transfer between bacteria, including distantly related taxa.

\section{REFERENCES}

Andrews S. C., Robinson A. K., Rodríguez-Quiñones F. 2003. Bacterial iron homeostasis. FEMS Microbiol. Rev. 27: 215-237.

ARnow L. E. 1937. Colorimetric determination of the component of 3,4-dihydroxyphenylalaninetyrosine mixtures. J. Biol. Chem. 118: 531-541.

BudZIKIEWICZ H. 1997. Siderophores of fluorescent pseudomonads. Z. Naturforsch. 52c: 713-720.

CsÁKY T. Z. 1948. On the estimation of bound hydroxylamine in biological materials. Acta Chem. Scand. 2: 450-454.

Dean C. R., Poole K. 1993. Cloning and characterization of the ferric enterobactin receptor gene (pfeA) of Pseudomonas aeruginosa. J. Bacteriol. 175: 317-324.

Dorsey C. W., Tomaras A. P., Connerly P. L., Tolmasky M. E., Crosa J. H., Actis L. A. 2004. The siderophore-mediated iron acquisition systems of Acinetobacter baumannii ATCC 19606 and Vibrio anguillarum 775 are structurally and functionally related. Microbiology 150: 3657-3667.

Faraldo-Gómez J. D., Sansom M. S. 2003. Acquisition of siderophores in Gram-negative bacteria. Nat. Rev. Mol. Cell Biol. 4: 105-116.

Haag H., Hantke K., Drechsel H., Stojilitikovic I., Jung G., Zähner H. 1993. Purification of yersiniabactin: a siderophore and possible virulence factor of Yersinia enterocolitica. J. Gen. Microbiol. 139: 2159-2165.

Hartmann H., Eltschig H. K., Wurtz H., Hantke K., Rakin A., Yazdi A. S., Matteoli G., Bohn E., Autenrieth I. B., Karhausen J., Neumann D., Colgan S. P., Kempf V. A. J. 2008. Hypoxiaindependent activation of HIF-1 by Enterobacteriaceae and their siderophores. Gastroenterology 134: 756-767.

Hope A. C. A. 1968. A simplified Monte Carlo significance test procedure. J. R. Statist. Soc. Ser. 30: $580-598$.

Karch H., Schubert S., Zhang D., Zhang W., Schmidt H., Ölschläger T, Hacker J. 1999. A genomic island, termed high-pathogenicity island, is present in certain non-O157 Shiga toxinproducing Escherichia coli clonal lineages. Infect. Immun. 67: 5094-6001. 
Lawlor M. S., O’Connor C., Miller V. L. 2007. Yersiniabactin is a virulence factor for Klebsiella pneumoniae during pulmonary infection. Infect. Immun. 75: 1463-1472.

Manninen M., Mattila-Sandholm T. 1994. Methods for the detection of Pseudomonas siderophores. J. Microbiol. Methods. 19: 223-234.

Meleney F. L. 1931. Bacterial synergism in disease processes. Ann. Surg. 94: 961-981.

Meyer J. M., Valerie A. G., Baida N., Gardan L., Izard D., Lemanceau P., Achouak W., Palleroni N. J. 2002. Siderophore typing, a powerful tool for the identification of fluorescent and nonfluorescent pseudomonads. Appl. Environ. Microbiol. 68: 2745-2753.

Mossialos D., Amoutzias G. D. 2008. Role of siderophores in cystic fibrosis pathogenesis: foes or friends? Int. J. Med. Microbiol. 299: 87-98.

Nelson A. L., Ratner A. J., Barasch J., Weiser J. N. 2007. Interleukin-8 secretion in response to aferric enterobactin is potentiated by siderocalin. Infect. Immun. 75: 3160-3168.

Okeke I. N., Scaletsky I. C. A., Soars E. H., Macfarlane L. R., Torress A. G. 2004. Molecular epidemiology of the iron utilization genes of enteroaggregative Escherichia coli. J. Clin. Microbiol. 42: 36-44.

Podschun R., Ullmann U. 1998. Klebsiella spp. as nosocomial pathogens: epidemiology, taxonomy, typing methods, and pathogenicity factors. Clin. Microbiol. Rev. 11: 589-603.

Rabsch W., Winkelmann G. 1991. The specificity of bacterial siderophore receptors probed by bioassays. BioMetals 4: 244-250.

ReIsSBRodt R., RABSCH W. 1988. Further differentiation of Enterobacteriaceae by means of siderophore-pattern analysis. Zbl. Bakt. Hyg. A. 268: 306-317.

Różalska B, Lisiecki P., Sadowska B., Mikucki J., Rudnicka W. 1998. The virulence of Staphylococcus aureus isolates differing by siderophore production. Acta Microbiol. Pol. 47: 185-194.

Schubert S., Picard B., Gouriou S., Heesemann J., Denamur E. 2002. Yersinia high-pathogenicity island contributes to virulence in Escherichia coli causing extraintestinal infections. Infect. Immun. 70: 5335-5337.

Schwyn B., Neilands J. B. 1987. Universal chemical assay for the detection and determination of siderophores. Anal. Biochem. 160: 47-56.

Visca P., ImPeri F., LAMONT I. L. 2007. Pyoverdine siderophores: from biogenesis to biosignificance. Trends Microbiol. 15: 22-30.

Vokes, S. A., Reeves S. A., Torres A. G., Payne S. M. 1999. The aerobactin iron transport system genes in Shigella flexneri are present within a pathogenicity island. Mol. Microbiol. 33: $63-73$.

Weaver V., Kolter R. 2004. Burkholderia spp. alter Pseudomonas aeruginosa physiology through iron sequestration. J. Bacteriol. 186: 2376-2384.

WeinBerg E. D. 1978. Iron and infection. Microbiol. Rev. 42: 45-66.

XIao R., Kisaalita W. S. 1997. Iron acquisition from transferrin and lactoferrin by Pseudomonas aeruginosa pyoverdin. Microbiology 143: 2509-2515.

Yамамото S., Oкujo N., SaKakibara Y. 1994. Isolation and structure elucidation of acinetobactin, a novel siderophore from Acinetobacter baumannii. Arch. Microbiol. 162: 249-254. 